\title{
Medical Genetics: Problems and Approaches
}

\section{Mohammad Saad Zaghloul Salem*}

Faculty of Medicine, Ain-Shams University, Cairo, Egypt

\begin{abstract}
Radical prevention of genetic diseases and genetically-determined defects, through efficient prophylactic and effective curative approaches, represents the final goal of medical genetics. However, success in achieving this objective is totally dependent on comprehensive understanding of all structural and functional aspects of the human genome, transcriptome and proteome. A lot of perplexing phenomena and enigmatic problems in medical genetics, as well as in human genetics, are awaiting this understanding to be disclosed, e.g. genomic imprinting, spontaneous mutation, evolutionary adaptations, apoptosis, carcinogenesis, functions of pyknons, significance of transposons, roles of micro RNAs and many others. Although the human genome project is steadily progressing towards full structural characterization of the human genome, a parallel human genome function project is indispensable for finalizing our knowledge of our genetic constitution. Similarly, human proteome structure and human proteome function projects are necessary for the same purpose. The extreme complexity of the structure of the human genome and human proteome necessitates new accurate analytical methods to study and understand the behavior and capabilities of both of these structured biosystems. These methods should rely on laws of thermodynamics, principles of quantum mechanics and, even, concepts of nanosciences. It is hoped that these new research approaches will help in revealing and defining the underlying mechanisms responsible for maintaining integrity, stability and identity of the genome and proteome which represent prerequisite knowledge needed for controlling and directing the functions of both of them for attaining optimal health and welfare of human beings. The present article shortly addresses some of the aforementioned problems and tries to offer few hypotheses, interpretations and proposals that might prove helpful in this respect.
\end{abstract}

Keywords: Genomic integrity; Genomic stability; Genomic identity; Evolutionary adaptations; Spontaneous mutations; Mitochondrial gene therapy; Chromosome transfer/removal therapy; Quantum genetics; Nanogenetics

\section{Problems and Approaches in Medical Genetics}

Although clinical aspects of medical genetics occupy the core of interest of physicians taking care of patients with genetic disorders, sound knowledge of basic aspects of human genetics, which represent the actual source of all information related to their work, is necessary for optimizing their practical skills. Unfortunately, a wide gap exists between clinical geneticists and required proper awareness of the spectrum of different fields of basic genetics. A situation like this might not hinder or affect the quality of medical service offered to patients, but it surely delays significant therapeutic and/or prophylactic achievements that could be attained by clinical geneticists familiar with basic genetics. For instance, knowledge of transposon activity and of unique features of mitochondrial genetics, when merged with relevant clinical data concerning development of congenital malformations and pathogenesis of mitochondrial disorders, could offer new research concepts regarding prophylaxis against congenital anomalies and therapy of mitochondrial disorders, respectively.

Although the human genome project is proceeding steadily towards full structural characterization of the human genome, absence of a parallel functional genome project represents a real challenge and constitutes a major obstacle to attain full functional characterization of the different components of the genome. Complete functional characterization of the genome is a prerequisite step necessary for complementing our current knowledge in different fields of medical genetics since genetic disorders are caused, primarily, by defective function(s) of genes following alteration(s) in their structure.

\section{Spontaneous mutation}

Occurrence of spontaneous mutations represents a major problem and real dilemma in medical genetics. In contrast to inherited diseases that can be anticipated, treated or alleviated, or even prevented altogether through preconception or prenatal diagnosis, genetically-determined disorders happening unexpectedly as a result of spontaneous mutations in offspring of apparently normal parents leave a very narrow margin and quite few options when counseling advice is presented to parents. Detectable fetal structural malformations caused by spontaneous mutations do not have the same unfortunate clinical impact since they are amenable to prenatal diagnosis, but genetic diseases caused by spontaneous mutations and characterized by defective functioning of one or more organs or body systems that can't be detected or diagnosed by currently available diagnostic techniques represent a real challenge to prophylactic measures aiming at reducing the burden of genetic diseases in populations.

Transposon-induced spontaneous mutation: The possible mechanisms underlying occurrence of spontaneous mutations need special emphasis, being the least defined among currently known subclasses of mutation. At least two possible potential causes of spontaneous mutations can be delineated: transposon activity and changes of energy equilibrium and dynamics of DNA. Transposon activity represents a major potential cause of spontaneous mutations because their movement results in insertional mutagenesis and

*Corresponding author: Mohammad Saad Zaghloul Salem, Professor of Medical Genetics, Faculty of Medicine, Ain-Shams University, Cairo, Egypt, E-mail: mszsalem@hotmail.com, mszsalem@yahoo.com

Received February 18, 2013; Accepted March 25, 2013; Published March 28 , 2012

Citation: Salem MSZ (2013) Medical Genetics: Problems and Approaches Human Genet Embryol S5: 002. doi:10.4172/2161-0436.S5-002

Copyright: ( 2013 Salem MSZ. This is an open-access article distributed unde the terms of the Creative Commons Attribution License, which permits unrestricted use, distribution, and reproduction in any medium, provided the original author and source are credited. 
disruption of targeted genes or other functional genetic elements. Disturbed and/or disrupted gene function with resultant deficiency of gene product or production of defective protein/RNA and suppression or interruption of genetic regulatory mechanisms exerted by mutated genes follows transposon-induced mutations.

Transposon activity might constitute one major source of spontaneous mutation in human genome. They, probably, underlie the pathogenesis and development of a wide range of congenital malformations due to their increased activity in the developing fetus, an effect that is counteracted by the silencing action exerted by piwiRNA on transposon sequences [1]. Available basic knowledge in this regard allows for drawing some suggestions that might prove useful in designing prophylaxis measures against development of some types of congenital anomalies. Construction of transposon maps of transposon elements, which constitute nearly $40 \%$ of the genome, seems mandatory for clarifying structural organization of the largest sector of the genome. Such maps would have great positive impact on many aspects of medical genetics. For instance, comparative analysis of these maps in normal fetuses or normal individuals, with those of fetuses or individuals affected with specific types of congenital malformations, through wide genome scans, might be helpful in disclosing some aspects of the cause and effect relationship between transposon activity and specific malformations. The effective opposition of this peculiar teratogenic mechanism by the silencing actions exerted by piwiRNA subclasses on transposon regulatory sequences, represents an important antimutation mechanism responsible for maintaining genomic integrity and genomic stability during this critical period of life. Comparison of malformation-specific transposon maps with their normal counterparts would offer invaluable information which can be used to construct anomaly-specific databases correlated with these particular transposon maps that depicts specific overexpressed transposons in specific types of congenital malformations. These data could have predictive value in delineating malformation-causing transposons, as well as potential therapeutic and/or prophylactic application by choosing and designing proper and specific piwiRNA silencing molecules against these particular anomaly-causing transposons.

Alternatively, creation of new genomic combinations between receptor segments of the genome and inserted transposons might be considered, within the context of evolutionary genetics [2] as one mechanism participating in genomic diversity and phenotypic evolution if these novel genomic combinations result in construction of new functional genetic elements and begin their own expression. Formation of new metabolic networks and establishment of new organized regulatory pathways are two possible mechanisms that can underlie the acquisition of new genetic phenotypes due to transposon activity. Validation of these hypothetical assumptions for the human genome, however, requires confirmation.

Disturbed DNA dynamics and spontaneous mutation: Double stranded DNA molecules behave like thermodynamically-stable structured system with assembling or binding energy larger than the potential thermal energy contained within its constituents. Nevertheless, changes of energy states of individual hydrogen bonds between complementary bases of the molecule can induce, and are potential causes of, spontaneous mutations. Alterations resulting from changes in electron resonance and/or proton transfer or relocation of bases or of the hydrogen bonds between them can lead to tautomeric shift or base tautomerization and momentary change of one base to another base, thus inducing point transition mutations [3]. However, the actual triggering mechanisms underlying the occurrence of these energetic changes of hydrogen bonds of bases of DNA are still largely undefined, precisely.

Changes of energy equilibrium and configurational dynamics of DNA as a whole might cause transient change of the molecule to a new structural configuration with different dynamic status. This change might pave the way towards development of pathogenetic alterations capable of causing spontaneous mutational events. The change of the structural configuration of DNA from the B form to $\mathrm{Z}$ form in acidic environments and the resulting propensity towards interruption of proofreading mechanisms controlling the replication of Okasaki fragments, thus leading to faulty and uncontrolled replication, might, possibly, be one underlying pathogenetic mechanism responsible for development of triplet repeat expansion disorders [4].

Atomic and sub-atomic dynamic changes of biomolecules in response to increased acidification of biological microenvironments under conditions of dehydration are well-known biochemical and pathophysiological alterations. The critical change of DNA structural configuration from the $\mathrm{B}$ form to $\mathrm{Z}$ form with consequent functional derangements induced by these biochemical alterations, happening at atomic level and governed by proton/electron energy states, adds further support to the need for studying the structure and functions of the genome and proteome according to laws and principles of quantum mechanics, which constitute efficient and exquisitely accurate analytical approaches towards disclosing many, still undisclosed, enigmatic aspects of medical genetics.

\section{Malignancy and the concept of genomic integrity}

Structural integrity of the human genome denotes presence of all its nuclear, mitochondrial and cytoplasmic membrane associated components in normal stable configuration. Maintaining stability, integrity and species-specific identity of the genome represents a prerequisite, not only for executing cell functions properly, but more fundamentally for initiation, continuation and conservation of life. Preservation of collaborative and integrated intimate relationships of these three aspects of the genome represents the real kernel and true essence of existence of all forms of life. Many pathogenetic mechanisms that drastically affect genome stability and genome integrity have been defined. These particular groups of mutations constitute important detrimental events that act via different pathways, e.g. defective genetic repair and premature induction of apoptosis, leading ultimately to intolerable loss of genome stability and integrity and induction of cell death. On molecular or cellular level, these mutations might be considered as lethal or life-ending mutations. These mutations might also act in a different way through total loss of reproductive fitness and disappearance of individual genomes from the gene pool of certain species.

However, the ability of malignant cells to develop marked selective advantages allowing them to survive and overcome normal cells, in a progressive vigorous behavior, in spite of extreme degrees of aneuploidy, e.g. hypodiploidy with 24 chromosomes, or near haploidy, [5] and hyperdiploidy with 74 chromosomes [6], which obviously represent eccentric states of genomic deficiency and genomic overgrowth, respectively, raises many assumptions regarding the classic concepts of genomic integrity and stability. Monosomy, or presence of one chromosome only in somatic cells instead of two, is incompatible with life except for $\mathrm{X}$ chromosome monosomy in female patients with Turner syndrome $(45, \mathrm{X})$. Autosomal monosomy has never been documented for sure in living subjects since normal embryonic and fetal development requires active participation of all chromosomes 
of the genome during this sensitive period of life. The contradiction between lethality of autosomal monosomies in somatic cells and vitality of malignant cells with marked hypodiploidy is quite puzzling and poorly understood since the wide spectrum of functional capabilities of the malignant phenotype requires over expression, rather than under expression, of most portions of the genome. Hypodiploidy of malignant cells results in corresponding deficiency of the proteome. Though quantitative proteome deficiency can be compensated for, within limits, by gene over expression and over transcription of mRNA with consequent increases in rate of translation, extreme hypodiploidy represents an incomprehensible state of genomic stability and proteome integrity.

This contradiction is further complicated by the close similarities between the behavior of malignant cells and early embryonic and fetal cells, notably reversion to undifferentiated totipotent stage. A major difference, however, resides in the finding that in early embryonic and fetal cells nearly the whole genome functions to synthesize, nearly, the whole proteome whereas in cancer cells with marked hypodiploidy fewer numbers of genes can synthesize a more efficient proteome with obvious selective advantage over normal proteome. The malignant phenotype is attributed to, and mediated by, oncoproteins synthesized by cancer cell genome. Their production by hypodiploid genomes can, at least theoretically, occur in view of many mechanisms that allow synthesis of multiple different proteins by one single gene, e.g. induced missense mutations of oncogenes, differential excision of introns and mRNA editing. Reversion to the embryonic stage seems to be a pivotal factor in maintaining the malignant phenotype initiated by overexpression of oncogenes because genomic functional potentials are optimally expressed during this stage. Similarly, synthesis of the full proteome is a characteristic property of embryonic and fetal cells. Clonal evolution of malignant cells depends, primarily, on genomic alterations expressed as different karyotypes of different malignant cells. This observation raises the query of whether it is justified to consider genomic integrity a cell-specific, rather than a speciesspecific, property inherited from the zygote and adapted according to the functions of the cell. It also raises another query of whether the acquisition of selective advantages of the proteome of malignant cells is a reflection of evolutionary adaptation of these cells. It might be more plausible to describe these changes of malignant cells as involutionary, not evolutionary, adaptations since they ultimately lead to death of the malignant cell or the whole organism with extinction of the genome from the gene pool of the environment.

The ability of cancer cells with different karyotypes to survive and proliferate more vigorously than normal cells, and the ability of differentiated normal somatic cells with different states of genomic activity, where each cell type has its own characteristic sets of active genes and of suppressed genes, to survive and perform its functions, raises the possibility of the aforementioned observation of whether genomic integrity is a cell-specific property characteristic of the genome of each cell. The strict synchronization, complementation and regulation of functions of innumerable numbers of cells in tissues and organs of humans as well as of other multicellular organisms, however, necessitates the presence of, still unknown and poorly defined, epigenomic master regulatory influences operatinground the clock in a continuous conclusive pattern in order to keep the genome, transcriptome and proteome identity of the organism. Full disclosure of these presumed epigenomic regulatory factors will probably, lead to better understanding of the pathogenetic mechanisms that underlie initiation of carcinogenesis, maintenance and progression of the malignant phenotype. Better understanding of these mechanisms would, surely, have great beneficial impact on predictive, prophylactic and therapeutic approaches in the fight against cancer.

\section{Genomic imprinting}

In spite of accumulating observations, imprinting remains to be one of the most puzzling phenomena in biology because of lack of sufficient information regarding the underlying mechanisms that mediate its effects and, also, due to the many species-specific and interspecies unrelated aspects and mechanisms of imprinting. Imprinting denotes differential expression of an autosomal gene according to its parent of origin, and in view of the wide spectrum of vital functions attributable to imprinting, it seems to be a global biological feature of all living organisms including humans, animals, flowering plants and microorganisms like bacteria and phages. The vast majority of imprinted genes in mammals have fundamental roles in embryogenesis and development of the placenta. Other imprinted mammalian genes are involved in post-natal developmental processes. Within a different biological context, for example in insects, imprinting plays a crucial role in sex determination where genomic imprinting of the whole paternal genome with consequent functional haploidy is a prerequisite for normal development into males.

Alternatively, in flowering plants imprinting occurs in the embryo as well as in embryo-nourishing tissues, the endosperm, and it has been suggested that imprinted genes might have a role in controlling the flow of nutrients from the mother to the offspring, a hypothesis referred to as the kinship theory. The observation that imprinted genes in flowering plants are located in the vicinity of transposable elements suggest that insertion of transposons is a prerequisite for imprinting of adjacent genes to control transposon activity in gametes, a hypothesis referred to as the defense hypothesis [7]. Liking imprinting to transposon activity, however, could not be accepted or justified unless solid evidence of parent of origin influence on transposon activity is clarified.

Though many additional hypotheses have been proposed to explain the biological aims and the evolutionary origins of imprinting, e.g. natural selection hypothesis and parental conflict hypothesis, imprinting in essence represents a programmed regulatory mechanism of the genome to harmonize functions and interactions of the large number of genes that constitute it, so that integrity and stability of the genome, hence genomic identity, is maintained, preserved and conserved in spite of the continuously ever changing proteome phenotype and proteome dynamics of living systems. If genes constituting a specific genome are imprinted, either expressed or silenced, without strict dominant genomic regulatory mechanism(s) controlling temporal aspects of their functions, widespread, mostly random and possibly conflicting, qualitative and quantitative changes of the proteome synthesized by that genome will disturb the stability and integrity of the genome, through many different mechanisms, leading ultimately to decadence and loss of the species-specific genomic identity that characterizes living organisms.

The dependence of imprinting on parent of origin of imprinted genes in humans undoubtedly denotes, as yet unidentified, critical X chromosome-related influence on mechanisms involved in initiating and maintaining imprinting, not only of the X chromosome itself as in Lyonization, but also for other imprinted genes. This influence, at least in part, might have its origin in the very early stage of embryonic development when both copies of the $\mathrm{X}$ chromosome are fully functioning. A presumed genomic imprinting regulatory region located on the $\mathrm{X}$ chromosome may be responsible for controlling and inducing genomic imprinting of the other $\mathrm{X}$, or all other $\mathrm{X}$ chromosomes in 
excess of one in abnormal conditions, as well as other genes intended to be imprinted anywhere on the autosomes, since there is no point in postulating that $\mathrm{X}$ inactivation and imprinting of autosomal or Y-linked genes are mediated by different mechanisms. This presumed imprinting region on the $\mathrm{X}$ chromosome may exert its regulatory role via synthesis of enhancer/silencer factors, including micro RNA subclasses.

Revealing actual pathogenetic mechanisms that underlie and mediate genetic imprinting would, surely, have great impact on many aspects of prophylactic and therapeutic genetics. For instance, selective imprinting of overexpressed oncogenes would constitute an effective risk-free treatment approach for cancer devoid of complications associated with other forms of cancer therapies. Similarly, disclosing imprinting mechanisms underlying Lyonization or inactivation of the $\mathrm{X}$ chromosome and defining causative factors responsible for its occurrence could be radical treatment approach for numerical chromosomal aneuploidies characterized by presence of extra chromosomal material, e.g. trisomy, tetrasomy or even triand tetraploidy. Therapeutic imprinting of extra chromsomes would surely have great impact in treatment of patients with these numerical chromosomal aberrations.

\section{Pyknons}

In contrast to the solid concepts of classic genes functioning in known traditional ways, the recent discovery of pyknons added new insights to the complexity of the functional organization of the human genome. Pyknons are short non-coding DNA sequences about 2022 nucleotides in length. They are widely distributed in the nuclear human genome in both the inter-genic and intronic regions of the genome, constituting about $1 / 6^{\text {th }}$ of the human genome. This makes them the most frequent, variable-length DNA sequence motifs in the human genomes. Pyknons have a remarkable degree of structural conservation. Their presence in the 3' UTRs untranslated regions of genes may indicate a potential regulatory role in post-transcriptional processing and modifications of mRNA. Though they do not share in either protein synthesis or RNA transcription, pyknons are functional genetic elements associated with mediation of specific biologic cellular processes. They are putative factors implicated in susceptibility to some common human genetic disorders and disturbed genomic regulation of function(s) of pyknons might underlie the development of this genetic susceptibility [8].

The considerable size of pyknons in the genome coupled with the intimate functional relationship between them and many subtypes of microRNAs suggest a pivotal role played by pyknons, probably as global regulators of gene function. Some unique sequences of human genomes are designed from series of short template octamer sequences which are embedded into pyknon's sequences and represented by hundreds (up to thousands) of copies in a genome. The assumed regulatory roles of pyknons might be exerted via different mechanisms. The small nucleotide number of pyknons, similar to the small nucleotide number of most microRNAs species when viewed in light of the parallel similarity between these two components of the genome as regards their magnitude and distribution, elicits some questions regarding their origin in the genome, and allows for hypothesizing the possibility that pyknons might represent non-classic genes or unique transcriptional units capable of directing and regulating synthesis of some regulatory microRNAs species needed for performing specific biological functions.

\section{Genetic Memory and Evolutionary Variation}

In spite of the exquisite ability of the protein translation system to recognize and decode the mRNA transcript and to harmonize the actions of tens to hundreds of factors involved in protein synthesis, it can't recognize changes of the original genetic code embodied within the codon sequence of the mRNA transcript. It decodes and recognizes triplets of bases, or codons, along the transcript without giving attention to whether they are comparable and complementary to the original sequences of the gene or not. It seems that the protein translation system has no prior memory to predict the validity of the codon sequence of the mRNA transcript in respect to both the gene sequence and the amino acid sequence of the protein. Though this apparent defect might be considered a prerequisite for evolutionary variation of protein phenotypes necessary for acquisition of new functional abilities, e.g. formulation and construction of new metabolic pathways or acquisition of favorable selective advantages [9], since it allows for synthesis of different new proteins, it is a major cause of pathogenesis of genetic defects due to absence of a translation proofreading and repair system comparable to those of DNA and mRNA repair systems.

Absence of genetic memory necessary for proofreading of translated proteins is enigmatic and bewildering in view of the prime and critical significance of the translation process, since the majority of genetic diseases result from synthesis of defective proteins or deficient synthesis of required proteins. Though it might be considered as a genomic regulatory mechanism allowing for selective pressure to proceed in favor of evolutionary variation, it results in marked pathological effects on the organism if newly synthesized proteins are structurally defective. This obvious contradiction between potential, possibly, favorable effects and actual degrading consequences on the genome poses many fundamental queries as regards putative epi genomic regulatory mechanisms responsible for maintaining integrity and stability of the genome, both of which are pivotal conservative features mandatory for keeping species-specific genomic identity of the organism. On the other hand, it raises many inquiries as regards the actual significance and true purpose of evolution since one of the most plausible and considerable definitions of evolution entail improving potentials, performance, capabilities and persistence of biological systems and living organisms. Absence of proofreading mechanisms of translation of the proteome might, accidentally, allow for evolutionary changes to take place, but it mostly causes damage to the proteome with consequent deterioration of biological capabilities and survival fitness of living organisms.

\section{Proteins as Separate Independent Biological Systems}

The currently adopted traditional dogma of molecular biology states that the genome controls synthesis of the proteome. This regulation is achieved either directly in case of viruses with positive strand RNA genomes or indirectly through the transcriptome. It also states that proteins are the actual mediators of all life activities in all living organisms. The stringent cause and effect relationship between genome mutation and proteome dysfunction drawn from this dogma holds true in most instances and represents a common final pathway underlying the development of the vast majority of currently defined genetic disorders.

The perplexing finding that some freshly, in vitro, synthesized polypeptide chains have an inherent ability of undergoing rapid and spontaneous folding into its native, thermodynamically stable structural conformation, and that they can, also, undergo some secondary structural configuration changes, however, raises many questions regarding its biological significance because folding of polypeptide chains is a highly collaborative and complex process involving many interactions between amino acid residues, and is attained through specific folding pathways. Accordingly, spontaneous occurrence of 
protein folding might be looked at as a pre-programmed behavior. The further discovery of the peculiar nature and behavior of prion proteins and their confusing ability to undergo inherent self-replication and propagation through inducing misfolding of normal properly folded proteins in infected cells, thus transforming them to misfolded prionlike proteins, raises more suspicions regarding the inherent ability of proteins to induce specific biological alterations in nucleic acids-free biosystems. No other biomolecules have this innate ability of selfreplication or have the potential of independent acquisition of advanced or complex structural configurational changes.

The aforementioned findings suggest that proteins, probably, constitute a separate biological system with an inherent ability of selforganization and self-replication. Additionally, they suggest that some proteins might be individually able to regulate and control some aspects of life activities in living organisms. Most importantly, they raise many queries regarding the completeness of the central dogma of molecular biology and its ability to explain and interpret unsolved problems in biology that are still waiting for disclosure.

The formal similarity in structural and functional organization between genes and proteins as regards: 1. the coded information embodied within the building units of each: nucleotides of genes versus amino acids of proteins, 2. the peculiar internal specific organization of each: promoters/exons/introns of genes versus essential functional/non-essential protein domains, 3. the proofreading and repair systems of each: DNA proofreading and repair systems of genes versus chaperones proofreading and repair systems of proteins, 4 . the liability to encounter changes in structural organization and functional capabilities by external factors: mutagens and mutation versus protein denaturing agents and protein denaturation, and most significantly: 5. the compliance of both to rules of natural selection and adaptive evolution, all point to pivotal roles played by proteins as fundamental biomolecules that can act individually as regulators of biological systems in other, still unknown, ways independent of the well-known established mechanisms dictated by the central dogma of molecular biology.

The similarities between proteins and catalytic RNA, or ribozymes, as regards catalytic activity, multiple domain configuration and complex folding into secondary structure of ribozyme-substrate complexes [10] raise many questions regarding possible phylogenetic relationship between genes, ribozymes and proteins. Ribozymes may represent an evolutionary link between the genome and the proteome. They might have been the original primary transcripts capable of integrating genomic and proteomic functions in one biomolecule, and their persistence might be attributed to their participation in performing many important functions, e.g. splicing of mRNA, biosynthesis of tRNA and translation of proteins [10]. Within the context of evolutionary divergence, consequent characterization of structural and functional features of mRNA as separate transcript biomolecules distinct from ribozymes may be looked at as a specialization process resorted to for optimizing gene function by constructing separate biosystem, viz. the transcriptome, specialized in, and responsible for, transfer of genomic information needed for protein synthesis. The significance of this presumed evolutionary process might be interpretable in view of tendency of biological systems to conserve their energy content to guard against degradation and decadence of its components. Additionally, it might, also, be explained by laws of thermodynamics since energy expenditure needed for performing multiple and different consecutive enzymatic reactions mediated by the ribozymes within active metabolic networks is considerably larger than energy needed by less energetic biomolecules like mRNA. However, hypothetical assumptions in this regard are purely theoretical. They need experimental testing and accurate validation by laws of quantum mechanics and principles of thermodynamics, as well.

\section{The Protein Code}

The specific sequence of certain amino acids within the protein domains might point to or represent what can be termed protein code responsible for determining the structure and function of the domain based on the specific amino acids composing the sequence and their specific arrangement within the sequence, in a way comparable to defining specific amino acids by specific codons in the gene. Extension of this assumption allows for postulating that the biological functions and physicochemical properties of each protein domain might be determined by the unique sequence structure of its amino acids, or its code information, so that peculiar arrangement of certain numbers of specific amino acids, arranged and aggregated in a specific sequence within the domain, might be responsible for determining specific structural and functional aspects of the molecule. Nevertheless, these assumptions which need much experimentation and testing, are mere hypothetical speculations based on the many biological phenomena that have not yet been explained fully by the traditional dogma of molecular biology, and on their ability to interpret some of the enigmatic aspects of these phenomena, at least in part, by reconsidering proteins as separate biological systems with inherent abilities similar to, but distinguished from, those of nucleic acids, and by revising our classic concepts of considering proteins as mere effector mediators of life activities synthesized under strict control of the genetic material.

\section{Regulation of Genomic Functions}

Currently defined functional sequences of the human genome, probably, represent a tiny fraction of the total genome size. However, this debatable observation does not state any more than we are still far away from full structural and functional characterization of our genome. The assumption that the major portion of the human genome has no definitive functions seems irrational and unacceptable in view of many factors headed by its incongruity with basic laws of thermodynamics. As referred to previously, energy conservation is a universal and fundamental biological principle shared by all forms of life and bio-molecules, and it would be insensible to waste much of the cellular energy produced via many energy-consuming processes to maintain a considerably huge structured bio-system like the DNA without optimal beneficial feedback to the cell. A helpful approach towards defining full functional potentials and capabilities of the genome might be attained through stepwise systematic delineation, characterization and classification of its different and varying structural components first, because structural characterization of biological systems is much more easier than revealing their functional potentials. A major drawback of the human genome project is absence of a parallel human genome function project aiming at depicting functional aspects of the structurally defined sequences and trying to correlate both aspects together in order to reveal the full structural-functional relationship and the essential proper framework of the genome. In spite of the desperate need for a human genome function project, a major theoretical obstacle to this objective, however, resides in the fact that the genome, as a structured biological system, is not an observable system because construction of the full framework of its internal status, i.e. complete functional potentials, can't be inferred from its observable output, i.e. currently defined proteome.

Defining the whole functional spectrum of the human genome is 
a prerequisite step necessary, not only for revealing and understanding regulatory mechanisms underlying genetic functions, but also for interpretation of some phenomena related to, and dependent on, regulation of the genome itself. The central dogma of molecular biology defines the intimate sequential relationship between the genome, the transcriptome, the proteome and life processes in the cell but it does not offer any assumptions or explanatory hypotheses as regards factors that regulate the functions of the genome itself. Maintaining genomic integrity, stability and identity is attained by many apparent genetic mechanisms, e.g. semi-conservative replication and DNA repair that are, in turn, regulated by specific components of the genome. This mutual functional interaction poses many questions and does not offer any clues as regards factors controlling functions of the genome and responsible for keeping its integrity. In this respect, irrational and injudicious Darwinian concepts stand helpless and profoundly unable to offer any answers to any of the relevant questions that are raised regarding these observations. Full comprehension of structural and functional aspects of the human genome will certainly lead to near complete understanding of pathogenetic mechanisms underlying pathogenesis of genetic disorders, thus helping in designing effective prophylactic and curative measures for management of these diseases. It will also help in revealing hidden mechanisms responsible for regulation of many, as yet, unexplained and mysterious genetic phenomena like genetic imprinting, apoptosis, metabolic adaptation and malignant transformation. On the long run, full structural and functional characterization of the genome will, undoubtedly, have a major impact on different aspects and approaches of eugenics.

\section{Genomic Adaptations to Stress and Evolution}

Reconsidering the proteome as being a separate and independent biological system participating in defining life framework of living cells, not merely a tributary structured system synthesized under regulatory control of the genome, might help in interpreting some perplexing aspects of evolutionary adaptation. Duplication of the genetic material, either on gene level or chromosome level, is looked at as evolutionary solution to stress and evidence of their occurrence in yeast has been revealed in many studies [11]. Increased genome size in response to stressful conditions, nevertheless, should be induced first by deficiency in proteome functions because stressful environmental stimuli exert their unfavorable impact first on the metabolic networks in the cytoplasm or in other cellular components. They do not affect the genome in a straightforward way except under certain extraordinary conditions, e.g. direct damage to genes. Neither qualitative nor quantitative changes of the proteome have direct or lasting effects on constitution of the genome in a manner capable of compelling the genome to respond in an adaptive way leading to duplication of some of its components. Compensatory increase of gene product in response to stressful conditions can be attained via increasing gene expression by many known mechanisms, e.g. enhancing transcription through promoter activation, increasing stabilization of mRNA and multiple translation rounds of the same transcript, without need to increase genome size by gene or chromosome duplication. Modifications of DNA-associated histones, for instance, have both enhancing and silencing regulatory effects on concerned genes but they do not result in, or induce genetic duplications or increase in genome size.

The concept of biological evolution stands in direct contradiction with our current knowledge regarding the structural and functional characteristics of both the genome and proteome. Stressful environmental conditions, traditionally considered as the main triggers of evolutionary adaptations, affect the proteome of the cell first. Accordingly, if assumptions regarding genomic adaptations as evolutionary processes are postulated, they have to reveal first how primary disturbances in structure or function of the proteome can lead to adaptive changes in structure and function of the genome. Though the possibility of formulating novel, meaningful metabolically active networks composed of, and mediated by, new functioning proteins synthesized as a result of defective translation, is extremely difficult to accept or to interpret by the basic concepts of randomness and/ or coincidence, its occurrence will be temporally limited by the life cycle of the cell. Unless adaptive genomic changes creating new genes coding for the new proteins occur, newly formulated networks can't be maintained, fixed or inherited. Some sort of interactive feedback mechanism between the proteome and the genome, similar to classic stimulus-response pathways, might exist and represent the missing link mediating this proteome-genome interaction. However, these assumptions rely, basically, on the hypothesis of participation of the proteome, as a separate and independent regulatory structured system, in conducting life processes in the cell and in inducing adaptive and sustained changes of the genome.

Alternatively, supporters of the theoretical hypothesis which postulates that evolution and divergence could be initiated, mediated and maintained, primarily, by genomic adaptation in response to stressful or demanding environmental conditions have to reveal induced mutagenic beneficial effects capable of causing increases in genome size. Currently known mutagens, e.g. irradiation-chemicalsviruses, are damaging agents and result in detrimental effects and pathogenetic consequences leading, in most instances, to disease. The main support to this hypothesis comes from findings indicating that a significant part of the human genome, making up nearly $8 \%$ of its size, is composed of endogenous retrovirus elements and fragments [12].

The mere presence of endogenous viral sequences in the genomes of higher species including humans, however, can't be considered neither as an indication of genomic evolution nor as a causative factor participating in its initiation or progression for many reasons:

First: infection of germinal cells involved in reproduction by the virus genome, a prerequisite step for transmitting the viral genome to offspring and fixing the new host-virus genomic recombination, is a rare event.

Second: efficient protective proofreading and anti-mutation mechanisms of the genome against unrecognized, or unregistered, sequences do exist and result in successful recognition of inserted strange viral genomes followed by their excision and deletion during genomic recombination stage via a specific mechanism known as recombinational deletion [13].

Third: with one exception only, HERV-K (HML2) gene family, no human endogenous retrovirus elements capable of replication have been identified; all studied elements appear to be structurally defective due to major deletions and/or nonsense point mutations [14].

Fourth: non-pathological insertional mutagenesis induced by viral sequences are expected to occur in intergenic, intronic or nonfunctional segments of the genome otherwise, they would result in decadence rather than evolution of the genome if they are inserted within functional sequences, e.g. exons, thus leading to their disruption. Complete physical mapping of all assumed endogenous retroviral elements is necessary to disclose this aspect of genomic adaptation if any roles in genomic evolution are attributed to these elements.

Fifth: the many regulatory roles attributed to these elements in 
controlling some of the most critical aspects of life activities of infected host genome, e.g. reproduction and immune competence, poses many questions regarding integrity and stability of the host genome before the acquisition of these elements. It is hard to accept the idea that a complex genome, like the human genome, would be pawned and dependent on external processes, like accidental insertion of endogenous retrovirus elements, to maintain its integrity, preservation and continuation through reproduction.

The concept of biological evolution needs reevaluation as regards some of its basic assumptions. Presence of similar, even identical, genetic sequences in different organisms irrespective of their phylogenetic positions, humans and drosophila for instance, needs not be an indication of anything other than those similar biological functions in living organisms are expected to be regulated by similar genetic sequences and mediated by similar proteomic networks. There is no logic in postulating that performing a particular physiological function in human cells necessitates more complicated genetic systems or more complex metabolic pathways than corresponding requirements for performing the same function in lower or any other species. Also, presence of endogenous retroviral elements in the human genome and their roles, not only as a subset of transposable elements but also as regulators of gene expression, could be interpreted in an equally plausible different contrasting way. Acquired elements, following viral infection and insertional mutagenesis, might have been used by the host genome for its own benefit to perform the functions attributable to these elements. Obviously, this explanation is more plausible than nonsense postulations attributing super capability and dominance of inserted viral genomes over infected human genome that enable it to induce evolutionary changes or directed adaptations in the human genome. Phylogenetic taxonomy of living organisms clearly discriminates between human beings, Homo sapiens, on one side and all other living organisms, viz. animals-plants-microorganisms including archaea, and reveals the uniqueness of human as distinct single species compared to thousands of species comprising each of the three other kingdoms of living organisms. The persistence of this characteristic phylogenetic distinction between humans and all other creatures withstands as coherent theoretical and logical obstacle against the core concept of evolution, and points to the existence and persistence of the human genome as a unique bio-system all through human life on earth.

In fact, logical analysis of the concept of evolution reveals, in a very obvious manner, that evolution is insensible idea. Postulations regarding final chance occurrence of self-assembly of biomolecules that started life activities, i.e. nucleic acids and proteins, after innumerable random reactions ignore the simple and firm fact that chance, by definition, has no memory. The Regular recurrence of biological phenomena in the same persistent, strict and repetitive pattern as seen in growth and development of living organisms, as well as in all their life activities, can never be attributed to chance and demolish these postulations. Regular recurrence of specific behavior patterns of biomolecules is solid indication of their being pre-programmed to behave in the same way under similar environmental effectors. Similarly, the construction of pre-programmed structured systems, like the genome, the transcriptome and the proteome, that obey definite physical laws and behave in accord to strict regulatory principles applicable to solid and living matter, as well, nullifies allegations as regards the ability of biomolecules to evolve, depending on innate self-assembly, in a selective pathway away from these laws. If we accept the idea that deeply seated roots of evolution began by chance event, then stochastic behavior of components undergoing evolution would be the rule in view of the continuous and persistent external effectors and stimuli they are exposed to. The exquisite control of structures and functions of biomolecules, living matter and living organisms throughout their life span invalidates any significance to these postulations regarding origin of life and evolution.

\section{Comparative Genomics}

The human genome is unique in being characteristic of one species only: Homo sapiens or humans. All human beings irrespective of their ethnic background have the same genome, albeit with minor differences. Conversely, animal, plant and microbial genera and species have different genomes characteristic of each species and shared, in many instances, by other subspecies of the same genus. The presence of innumerable numbers of genetically distinct living species, other than humans, without any genetic evidence of changes attributable to evolutionary adaptation should compel us to reconsider the unjustified tendency for applying results of research and experimentation on these species to humans. What might be considered as evidence of evolutionary adaptations, e.g. development of novel metabolic circuits by some microorganisms in response to environmental effects, might be due to activation of already existing, still undefined and unrecognized, genes or other functional components of the genome responsible for mediating these novel functions under the influence of the new environmental conditions. The frequent delineation of new adaptive metabolic networks without defining new genes responsible for their establishment stands in favor of this interpretation. Obviously, unless new genes responsible for synthesis of new proteins mediating these novel networks are mapped, defined and characterized for sure, these metabolic adaptations could never be considered within the context of evolution.

Although the development and appearance of new biological functions secondary to environmental effectors or endogenous mutational events reflects the concept of adaptation, a fundamental and universal biological principle shared by living organisms and indispensable for any biological systems existing in a continuously varying and stressful environment, adaptation needs not be considered as modifications secondary to genomic alterations. As referred to previously, the concept of evolution is hard to consider unless solid evidence of progressive and appreciable increase in proteome size accompanied by parallel increase in genome size from simple to more complex biological systems, e.g. from unicellular to multicellular organisms, is provided. Actually, this is not the case as many simple organisms have larger genomes than more complex organisms.

The human genome, like genomes of other living creatures, is programmed in a very strict way to behave in a very defined manner under specific recurring circumstances, for example replication during cell division and differential mass suppression and activation of many of its components during embryogenesis. Success in discovering and revealing underlying epigenomic, rather than epigenetic, factors and mechanisms responsible for regulating and maintaining the three fundamental aspects of the genome, viz. integrity-stability-identity, would, surely, have revolutionary impact on all aspects of human genetics and in particular medical genetics. However, the benefits of such an achievement are conditioned by their applications in different fields of medical genetics; otherwise they will probably lead researches to nothing except getting things back to old futilitarian nonsense discussions concerning adaptation and evolution. Within the context of medical genetics, researches aiming at revealing how things happen, in order to direct their happening for the welfare of patients, are much more important and cost-effective than wasting efforts, time and money in research studies trying to know why they happen. 


\section{Quantum Genetics and Nano-Genetics}

Analysis of DNA base behavior according to principles of quantum mechanics and quantum dynamics, rather than of classic Newtonian principles or even crude principles of thermodynamics, seems more appropriate and is essential for clarifying many obscure phenomena concerning structure and function of the genome, e.g. mechanisms of spontaneous mutation and of action of helicases. The concept of quantum genetics, where behavior of the genetic material is interpreted by principles and rules of quantum mechanics [15], would certainly prevail in the coming era due to progressive increase in our knowledge of the structure and function(s) of our genome and our proteome. Quantum genetics might even be exceeded by nano genetics, a term coined to signify the study of behavior of subatomic components of nucleotides at a nanoscale level, because many significant pathogenetic mechanisms that result in pathophysiological alterations leading to pathogenesis of genetic disorders are not interpretable except by laws that govern the behavior of matter at electron-photon level. Some structural and functional aspects of both the genome and the proteome even need more analytical approaches, e.g. principles of plasma physics.

The differing behavior of matter based on its structural state, whether as aggregated units or as single atoms, represents the core concept of nano sciences. Nano genetics imposes real need for formulating theoretical principles of nano sciences in medical genetics, not only for understanding and interpreting unexplainable phenomena of genetic concern, but, more importantly, for optimal use of applications of nanobiotechnology in diagnosis, prophylaxis and treatment of genetic diseases. The amazing invention of active biomolecules, biosensors, nano channels and self-assembling macromolecules, like nucleic acids, proteins and lipids, are just few obvious examples of the great potential of nanobiotechnology in medical genetics. Many categories of genetic disorders, for instance chanellopathies, membrane defects, storage diseases and protein misfolding disorders, would, probably, become amenable to treatment, even full cure, in the near future in light of these inventions based on nano sciences and nanobiotechnology.

\section{Formal Genetics: Future Prospects in Medical Genetics}

The definition of formal genetics is still a matter of contention. However, it can be defined as a branch of basic genetics concerned with deducing and figuring out relevant genetic data from constructed figures that contain specific genetic information. These informative figures include, for instance, constructed family pedigrees, linkage maps, restriction fragment length polymorphism maps and chromosomal maps. Though formal clinical maps, composed of pathognomonic combinations of signs, symptoms and specific pathological findings pointing to specific disease entities, represent the simplest of formal genetic maps they are not considered, strictly, of formal genetic concern. However, clinical maps when analyzed with other informative maps like linkage maps and chromosome maps, have major diagnostic importance in identifying significant data regarding the localization of undefined genes underlying, and possibly involved in, pathogenesis of certain disorders and malformations. Many aspects of applied and clinical genetics have been revealed in view of the information databases collected from studies of formal genetics of certain diseases and of specific experimental researches, e.g. construction of chromosomal maps of gene loci based on information gathered formerly from humanmouse hybridization studies, and currently by in situ hybridization experiments. Also, progress in disclosing and revealing interspecies genetic similarities and dissimilarities, which constitute major fields of research of comparative and evolutionary genetics, depends largely on species-specific genetic databases gathered and analyzed within the framework of formal genetics.

\section{Exome maps}

Progress in analysis of structural organization of the human genome generates a flood of information leading to characterization of new formal maps of specific DNA markers and regions of both structural and functional significance. These maps represent bioinformatics databases that can have crucial impact on many aspects of basic as well as of clinical medical genetics. For instance, exome maps comprising detailed information of exons of genes can be constructed and used for both intra and inter-species comparative purposes. Similarly, comparison of exome maps of patients with specific genetic disorders with those of normal subjects represents a promising approach that can have many diagnostic applications in clinical genetics. Other types of molecular maps that can be constructed based on available and rapidly accumulating databases of human genome structure, e.g. introme, pyknon, transposon, telomere and pseudogenes maps, can also have a wide spectrum of applications in many fields of medical genetics.

\section{Functional genomic maps}

The significant and beneficial applications of structural genetic maps in different fields of medical genetics call for construction of parallel functional genetic maps that characterize critical functional markers and transcriptionally active domains of the human genome. Hypothetical applications of functional genomic maps can include a wide range of prophylactic, diagnostic and therapeutic approaches. For instance, anticipation of possible complications in patients suffering from specific genetic disorders, and of possible health hazards in individuals with susceptible genetic background, based on formal genetic databases of their genomic and proteomic constitution, represents a great prophylactic approach with favorable prognostic prospective in management of patients with genetic diseases and their concerned family members. Also, it has considerable beneficial implications upon offering proper counseling advice to genetically susceptible individuals.

\section{Proteome maps}

Construction of comprehensive structural proteome maps comprising both structural and catalytic proteins, or enzymes, could have far reaching beneficial impact on many aspects of therapeutic genetics. Comparative analysis of proteome maps in normal and in subjects affected with specific genetic disorders can identify diseasespecific defective/deficient proteins responsible for pathogenesis of these disorders. This diagnostic approach might prove particularly helpful for idiopathic genetic diseases, the causative genes of which are not yet identified. It can also help in designing reasonable treatments based on detected proteomic changes, e.g. offering identified deficient/ defective proteins to patients affected with these disorders.

Comparative analysis of proteome maps of normal cells and of malignant cells can, also, depict functional deviations of the genome in cancer cells and detect oncogenic proteins synthesized and expressed by the malignant cell and responsible for pathogenesis and evolution of the malignant phenotype. Oncoprotein maps would, certainly, have beneficial impact on research studies aiming at better, and proper, understanding of malignant transformation through targeting pivotal oncoproteins that initiate and promote the malignant phenotype of cancer cells. They would, also, have similar impact on research studies aiming at designing and tailoring specific therapeutic trials for challenging carcinogenesis through e.g. engineering of monoclonal 
antibodies against relevant oncoproteins inside intracellular compartments as well as on cell membranes and within intercellular spaces. Interruption of signaling pathways involved in mediation of many selective advantageous functions of malignant cells, e.g. enhanced glycolysis, metastasis and neovascularization which depend on synthesis of specific oncoproteins could, probably, represent a promising approach in treatment of cancer through disassembly of the metabolic and regulatory networks actively participating in malignant transformation and evolution of the malignant phenotype.

\section{Transcriptome maps}

On molecular level, comparative analysis of transcriptome maps of messenger RNA and of different micro RNA species in normal and in diseased subjects could offer remarkable help in diagnosis of genetic diseases characterized by substantial genomic activities. Malignant cells express large numbers of actively transcribed genes and detailed cancerspecific transcriptome maps could be constructed for pathologically distinct types of malignancies, thus allowing for better understanding of the aberrant phenomena of differential suppression of certain genes and of mass overexpression of specific gene families, e.g. proto-oncogenes, in malignant cells. They can also offer more information on the genic and inter-genic pathogenetic and pathological mechanisms underlying development of specific types of cancer. Cancer-specific transcriptome maps and oncoprotein maps constructed for pathologically distinct types of malignancies might offer rapid and accurate diagnostic tools of these malignancies when compared with corresponding maps of normal cells. Delineation of the whole spectrum of proto-oncogenes and oncogenes in the human genome and construction of accurate informative maps detailing relevant databases of this spectrum remains, and represents, an ultimate objective and indispensable goal crucial for understanding and designing radical genetic therapies for cancer.

During embryonic and fetal development, and to much lesser extent in early post-natal life, organ and tissue-specific differential suppression of large numbers of certain sets of genes, paralleled by selective expression and/or over-transcription of similar numbers of other sets of genes, is mandatory for normal processes of specialization, growth and development. Formal analysis of databases of proteome and/or transcriptome maps during different periods of fetal development could reveal the intimate correlations between temporal and regulatory influences during each of the consecutive stages of development and reveal many obscure aspects of this critical period of life. This information might prove helpful in understanding genomic and proteomic disturbances that predispose to the pathogenetic mechanisms and the pathophysiological alterations that underlie the development of congenital malformations. Comprehensive understanding of these aspects of development represents a prerequisite step indispensable for hypothesizing and designing effective prophylactic measures and early therapeutic intervention approaches against teratogenesis.

\section{Transposon maps}

Formal analysis of databases of structural and functional genomic and proteomic maps of fetal development can add, significantly, to our current vague and scanty knowledge of many, still, unknown aspects of regulatory genetic mechanisms, particularly those of genetic and genomic imprinting and temporal synchronization of mass suppression and activation of large numbers of genes that characterizes development and differentiation. The role played by increased transposon activity in causing, seemingly, spontaneous mutagenic events that predispose to developmental malformations during fetal development deserves more attention if effective prophylactic anti-teratogenic measures are to be hypothesized. Also, the effective opposition of this teratogenic mechanism by the silencing action exerted by piRNA on transposon regulatory sequences, represents an important regulatory mechanisms responsible for maintaining genomic integrity and genomic stability during this critical period of life. Comparison of malformationspecific transposon maps with their normal counterparts would offer invaluable information which can be used to construct anomalyspecific databases correlated with these particular transposon maps that depicts specific overexpressed transposons in specific types of congenital malformations. These data could have both predictive value in delineating malformation-causing transposons, as well as potential therapeutic and/or prophylactic application by choosing and designing proper and specific piRNA silencing molecules against these particular anomaly-causing transposons.

Computational analysis of bioinformatics databases represented by different types of formal genetic maps, whether for comparative or predictive purposes, represents a real challenge. Not only because of lack of many pieces of information necessary to complete these maps, but also due to paucity of our knowledge as regards basic essential genetic mechanisms that regulate the structural integrity and functional specialization of the genome as a whole. For instance, the nature of the regulatory systems that master the highly conserved species-specific genetic constitutions of all creatures, including the human genome, is extremely vague, without any clues to the possibility of disclosing the design or the components of the genetic material responsible for it, even in the near future. Similarly, the significance of many puzzling and enigmatic phenomena regarding currently known details of structure and functions of the genetic material continues to be a matter of debate in spite of the many theories and hypotheses trying to explain them. Examples of such phenomena include genetic imprinting, transposon activity, apoptosis, evolutionary adaptations and many others. However, the mechanism through which solid biomolecules, notably nucleic acids and proteins, can conceive, interpret and execute pre-programmed biological processes that are embodied within their structural configuration remains on the top of these unsolved phenomena.

\section{Genetic Therapies: Problems and Proposals for New Frontiers}

Genetic diseases are caused by genomic errors, but are mediated by proteomic defects. The classic cascade of events leading to development of genetic diseases begins with spontaneous or induced mutational events causing structural alterations followed by functional defects of mutated genes. This pathogenetic mechanism, applicable to mutations of structural genes, results in improper transcription of mRNA and faulty translation with consequent synthesis of defective proteins or deficient synthesis of proteins. Alternatively, mutations affecting regulatory genes can lead to regulatory disturbances secondary to deficient or defective synthesis of transcription factors, regulatory RNA subclasses or regulatory nucleoproteins. These regulatory defects express themselves in many different ways like suppression of function of structural genes, defective post-translation modifications of proteins or protein targeting-trafficking defects. In either case, proteomic defects result in disturbed functioning of one or more metabolic/regulatory/ signal transduction networks thus leading to development of diseasespecific pathophysiological alterations that predispose to pathogenesis of disease and development of pathognomonic phenotype. This cascade reveals that genetic disorders ultimately develop in response to proteomic disturbances. Accordingly, it would be more logical, and more profitable, to target the proteome, not the genome, in genetic 
therapy trials. This rationale is, already, adopted successfully since current therapies of genetic disorders depend largely on approaches aiming at reversing proteome defects, e.g. supply of deficient proteins, replacement of defective proteins and inducing required alterations in proteome-dependent networks through use of drugs. However, there are too many considerable drawbacks of this therapeutic approach headed by the fact that it can't offer cure of genetic diseases, rather it provides temporary alleviation of sufferings and slowing of development of complications, so it has to be resorted to, regularly, for life. Another major drawback of proteome manipulation is reflected by the very wide spectrum of complications and side effects of drugs used for this purpose.

Analysis and interpretation of biological phenomena occurring within the framework of exceedingly active and reactive bioenvironments, e.g. nucleic acids and proteins inside the cell nucleus-the mitochondria and the cytoplasm, are mandatory to fully understand and define all structural aspects and functional capabilities of our genome-transcriptome and proteome. This comprehensive knowledge, in turn, is a prerequisite for designing effective treatments, even cure, of genetic disorders. Nevertheless, the marked complexity of the human genome, combined with lack of enough data regarding its structural organization and functional capabilities, reveals the potential hazards that might be caused by erroneous, injudicious or hypothetically-based manipulation of one or more of its components. Lack of comprehensive information of the genome represents an aborting challenge to single gene therapy trials aiming at repair or replacement of defective genes. The challenge stems from the fact that targeted genes are located within thousands of other genes and dispersed within an extremely condense environment of chromatin. In addition, and more importantly, these genes are tightly controlled by regulatory mechanisms exerted by other genes and perform specific functions within a genomic framework responsible for maintaining genomic integrity, genomic stability and genomic identity as well. Stem cell therapy, in essence a polygenic or a whole genomic therapeutic approach, faces less obstacles than single gene therapy trials but is hindered by other considerable theoretical difficulties. The significant structural and functional changes that are continuously imposed on the genome in a progressive manner during transition from embryonic-fetal stage to postnatal-adult stage, e.g. programmed tissue-specific suppression and/or activation of considerable portions of the genome, occur in response to, and are mediated by, extremely large numbers of temporally controlled, stagespecific and tissue-specific metabolic and regulatory networks working in strict harmony and optimal synchronization to guarantee normal development. Differentiation and specialization of stem cells in the embryo and the fetus occur within this extremely complex biochemical environment. The classic cascade of life processes comprising growth, differentiation, specialization and development that characterizes this critical and sensitive period of embryonic-fetal development necessitates full active participation of nearly the whole genome, transcriptome and proteome in establishing and regulating the huge number of networks responsible for its mediation. This complex situation seems impossible to be repeated or even near simulated, under any circumstances, within cellular and tissue microenvironments of post-natal life. In view of these difficulties, there is a real need for exploring other safer and more appropriate approaches to genetic therapies.

\section{Mitochondrial gene therapy}

The peculiar features of mitochondrial genetics and mtDNA molecules make it a plausible target for gene therapy trials. It also has many obvious advantages over classic gene therapy trials aiming at repair or replacement of mutated nuclear genes. The reasons for this assumption, which needs validation through much experimentation, can be summarized in the following notions:

1. The presence of mtDNA in the cytoplasm away from the nucleus obviates the known hazards associated with classic gene therapy trials directed towards nuclear genes, in particular random uncontrolled insertional mutagenesis, which results in widespread damage to many components of the nuclear genome.

2. The autonomous nature of mitochondrial replication allows for higher, more persistent and sustained production of the desired gene product due to the presence of many, hundreds to thousands, mitochondria in each cell and presence of multiple copies of mtDNA within each mitochondrion.

3. If viral vectors are used for DNA delivery, viral genomes do not replicate within the mitochondrion due to absence of other genes necessary for their replication, thus almost all of the deleterious consequences of viral replication within host cells and spread to other cells are avoided.

4. The presence of large copy number of mtDNA within each mitochondrion permits a safer margin in case of failure of gene delivery or damage to the mitochondrial genome since no genetic deleterious effects happen in living cells unless nearly $80 \%$ of its mitochondrial DNA content becomes mutated or malfunctioning.

5. Use of non-viral vectors, e.g. DQAsomes, gene delivery system made from Dequalinium [16], for gene delivery to the mitochondria, avoids all risks known to be associated with use of viral vectors for gene delivery.

\section{Correction of chromosomal aneuploidy}

The invention of pre-implantation diagnosis technique to avoid conception with embryos affected with single gene disorders represents great achievement as a prophylactic approach for this category of genetic diseases. Extension of the basic concept of this diagnostic approach might prove useful in treating, even curing, some types of numerical chromosomal aberrations, bearers of which have no reproductive options except of having chromosomally abnormal offspring, e.g. parent with 21/21 translocation. Carrier couples at possible risk of having offspring with monosomy or trisomy can be managed along the same, albeit modified, management protocols of pre-implantation diagnosis adopted and applied for those at risk of having offspring with single gene disorders. However, micromanipulation of the early embryonic stages seems more difficult, since all cells have to be manipulated, and proper intervention has to be performed better at the earliest zygote stage.

In vitro fertilization for couples having no reproductive options except of having chromosomal trisomies, and micromanipulation of trisomic zygotes for removal of the extra chromosome followed by implantation of normally treated zygotes in the uterus, seems to be plausible therapeutic approach, even, straightforward final cure of this category of numerical chromosomal abnormalities. In parallel ways, addition of normal individual chromosomes, isolated from one of the parents, to monosomic zygotes to replace for the missing chromosome, represents the main frame of the technical aspects of this proposal that might have a role in prophylactic treatment or cure of these specific types of chromosomal disorders, which have no cure up till now. The underlying theoretical postulations of these proposed 
technique comprising chromosome transfer and chromosome removal for treatment of numerical chromosomal aberrations, however, are confronted with many theoretical obstacles stemming, primarily, from disturbances of genomic regulatory processes that are expected to follow early manipulation of the genome. Ensuring normal functional integration of transferred chromosomes within the functional framework of the host genome is critical for regaining full functional potentials of treated monosomic zygotes. Also, strict precautions necessary to avoid the possibility of causing uniparental disomy is another technical difficulty in the procedure that necessitates delicate and accurate manipulations, otherwise pathogenetic burdens of some autosomal recessive disorders would be imposed on the treated zygote. Application of this hypothesized pre-implantation prophylaxis approach is apparently easier, and more safe, for monosomic defects since chromosome transfer can be performed at a post-zygotic, 8-16 cell stage embryo, stage. Though this research hypothesis seems, at least theoretically, possible and plausible prophylactic/therapeutic approach for these types of chromosomal abnormalities, it needs proper validation by tedious experimental researches.

Finally, it is hoped that completing and finalizing our current knowledge of the master regulatory mechanisms responsible for maintaining and preserving integrity, stability and identity of the human genome would have a great impact on all fields of medical genetics. For instance, as referred to previously, revealing regulatory mechanisms responsible for programming and controlling genomic imprinting and characterization of genes, microRNAs and proteins involved in mediating these mechanisms would offer final cure for many types of chromosomal aberrations, e.g. trisomies, through inducing imprinting of the extra chromosome(s). Similarly, imprinting of oncogenes would avail an ideal therapeutic approach for cancer. Designing transposonspecific piwiRNA can appreciably reduce incidence of transposoninduced congenital malformations as well as many other single and polygenic disorders caused by this pathogenetic mechanism.

It is also hoped that ultimate interpretations of genome behavior according to laws of quantum dynamics and principles of nano sciences, would offer correct answers to current enigmatic questions in genetics. It will also avail proper solutions to perplexing and unsolved problems in medical and human genetics, and genetics of other living organisms, as well. Concomitant invention and application of accurate diagnostic techniques, efficient prophylactic approaches and curative therapies for genetically-determined diseases, based on these interpretations, would then represent the final true success looked for in medical genetics.

\section{References}

1. Sienski G, Dönertas D, Brennecke J (2012) Transcriptional silencing of transposons by Piwi and maelstrom and its impact on chromatin state and gene expression. Cell 151: 964-980.

2. Slotkin RK, Martienssen R (2007) Transposable elements and the epigenetic regulation of the genome. Nat Rev Genet 8: 272-285.

3. Irwin H. Herskowitz (1977) Principles of genetics. Collier Macmillan International Editions., 2nd ed, pp. 135-152.

4. Bidichandani SI, Ashizawa T, Patel PI (1998) The GAA triplet-repeat expansion in Friedreich ataxia interferes with transcription and may be associated with an unusual DNA structure. Am J Hum Genet 62: 111-121.

5. Heerema NA, Nachman JB, Sather HN, Sensel MG, Lee MK, et al. (1999) Hypodiploidy with less than 45 chromosomes confers adverse risk in childhood acute lymphoblastic leukemia: a report from the children's cancer group. Blood 94: 4036-4045

6. Chng WJ, Kumar S, Vanwier S, Ahmann G, Price-Troska T, et al. (2007) Molecular Dissection of Hyperdiploid Multiple Myeloma by Gene Expression Profiling. Cancer Res 67: 2982-2989.

7. Köhler C, Weinhofer-Molisch I (2010) Mechanisms and evolution of genomic imprinting in plants. Heredity (Edinb) 105: 57-63.

8. Glinsky GV (2009) Human genome connectivity code links disease-associated SNPs, microRNAs and pyknons. Cell Cycle 8: 925-930.

9. Fletcher GL, Hew CL, Davies PL (2001) Antifreeze proteins of teleost fishes Annu Rev Physiol 63: 359-390.

10. Butcher SE, Heckman JE, Burke JM (1995) Reconstitution of hairpin ribozyme activity following separation of functional domains. J Biol Chem 270: 2964829651.

11. Yona AH, Manor YS, Herbst RH, Romano GH, Mitchell A, et al. (2012) Chromosomal duplication is a transient evolutionary solution to stress. Proc Natl Acad Sci U S A 109: 21010-21015.

12. Belshaw R, Pereira V, Katzourakis A, Talbot G, Paces J, et al. (2004) Long-term reinfection of the human genome by endogenous retroviruses. Proc Natl Acad Sci U S A 101: 4894-4899.

13. Belshaw R, Watson J, Katzourakis A, Howe A, Woolven-Allen J, et al. (2007) Rate of recombinational deletion among human endogenous retroviruses. $J$ Virol 81: 9437-9442.

14. Ruggieri A, Maldener E, Sauter M, Mueller-Lantzsch N, Meese E, et al. (2009) Human endogenous retrovirus HERV-K(HML-2) encodes a stable signal peptide with biological properties distinct from Rec. Retrovirology 6: 17.

15. McFadden J, Al-Khalili J (1999) A quantum mechanical model of adaptive mutation. Biosystems 50: 203-211.

16. Weissig V, Lasch J, Erdos G, Meyer HW, Rowe TC, et al. (1998) DQAsomes: a novel potential drug and gene delivery system made from Dequalinium. Pharm Res 15: 334-337. 